\title{
A methodology for solving facility layout problem considering barriers - genetic algorithm coupled with A* search
}

\author{
Mariem BESBES ${ }^{1,2^{*}}$, Marc ZOLGHADRI ${ }^{1}$, Roberta COSTA AFFONSO ${ }^{1}$, \\ Faouzi MASMOUDI ${ }^{2}$, Mohamed HADDAR ${ }^{2}$ \\ ${ }^{1}$ Quartz-Supmeca, 93407 Saint-Ouen, France \\ Tel: (+33)149452900; e-mail: (\{mariem.besbes, marc.zolghadri, roberta.costa \}@ supmeca.fr). \\ ${ }^{2}$ University of Sfax, LA2MP-ENIS, B.P. 1173, 3038 Sfax, Tunisia \\ e-mail: (\{faouzi.masmoudi, mohamed.haddar\}@enis.tn). \\ * Corresponding author
}

\section{INTRODUCTION}

Facility Layout problem aims to place a set of facilities on a given area so that certain criteria are optimized. The most efficient layout can contribute to the overall efficiency of the operations and can reduce about 20-50\% of the final operating cost [Tarkesh et al. 2009]. The facility layout problem (FLP) has broad applications in real life situations including layout of schools, hospitals and manufacturing systems [Ahmadi et al.2017]. In this study, we will interested by manufacturing perspective. The FLP can also be classified depending on the representation method. Generally, there are two representations [Tompkins et al., 2010]: (i) discrete representation, (ii) continuous representation. The discrete layout problem is the traditional approach to the FLP. It aims to place equal area facilities having rectangular shape in a predetermined locations [Chraibi et al. 2014]. However, this discrete approach to FLP is even more unsuitable when facilities have unequal-area and shape or if there are different clearances between the facilities [Drira et al. 2007].The existing FLP researches fall into several categories according to various criteria [Che et al. 2016]: equal-sized and unequalsized FLPs [Azadeh et al. 2016], static and dynamic FLPs [Moslemipour and Lee 2012], single-floor and multi-floor layouts [Ahmadi et al. 2016], single-objective and multi-objective problems [Samarghandi et al. 2010].

Resolution of the facility layout problem (FLP) is based either on exact methods to reach optimal solutions, or heuristics and meta-heuristics to get near-optimal solutions. Exact methods cannot give optimal solutions for larger-size instances due to the NP-completeness of such FLPs. For this reason, heuristics or meta-heuristics are the most appropriate methods for solving the large size of this problem in a reasonable computational time to offer near optimal solutions. These meta-heuristics include Genetic Algorithm (GA), Tabu Search (TS), Simulated Annealing (SA), Ant Colony Optimization (ACO) and Particle Swarm Optimization (PSO). Interested readers can refer to Kundu and Dan (2012) for an in-depth analysis of the different metaheuristics methods applied in FLPs.

Our main motivation is to explore a more relevant way to find the most efficient layouts with a satisfactory MHC considering constraints that are more realistic. Most of the FLP resolution approaches use the rectilinear or Euclidean distance between facilities [Gonçalves et al. 2015; Xie et al. 2018]. These distances can miscalculate the actual traveled distance in the case of restricted workshop. For this reason, Authors propose a new methodology and mathematical formulation to answer the constrained Facility Layout Problem. The main idea is first to use the $\mathrm{A}^{*}$ algorithm to identify the shortest path between equipment's in a more realistic way; in a facility area which contains obstacles and transportation routes. A Genetic algorithm is adapted and intertwined with $A^{*}$ algorithm to generate various candidate layouts. We study the effects of the GAs parameters on the quality of layout generation by using Monte Carlo Simulations. The best of class layouts obtained for every step of the simulation are collected 
and studied to find out the most interesting set of parameters and operators for the GA. The obtained facility arrangements are deeply discussed based on their material handling cost. The quality of the found arrangements obtained by using $\mathrm{A}^{*}$ algorithm is compared to the use of Euclidian and rectilinear distances. The mix of GA and $\mathrm{A}^{*}$ gives better layouts.

\section{PROBLEM FORMULATION}

The problem is to assign " $\mathrm{m} "$ machines in a given space in order to minimize transport costs between different machines. We define our workshop as a two-dimensional grid consisting of a set of units called non-transformable units. The workshop has a rectangular shape with predefined dimensions: a length (L) and a width (W). In addition, there is a defined horizontal corridor for the permanent transport of products. This corridor is characterized by two vertical positions or two limits $\left(y_{\text {lower }}\right)$ and ( $\left.y_{\text {upper }}\right)$ that divide the workshop into two sections. We also find obstacles such as walls, stairs, other barriers or corridors that can not be occupied by a machine. The coordinates of each obstacle are defined by $\left(x_{o}, y_{o}\right)$. The machines have a rectangular shape and have the same size, they are defined by the horizontal $\left(l_{i}\right)$ and vertical dimensions $\left(w_{i}\right)$ and the coordinates of the centroid $\left(x_{i}, y_{i}\right)$, see figure 1 . Among the constraints to be respected, we can mention that the assignment of the machines is done within the limits of the workshop, another set of constraints prevent the overlaps between the machines and guarantee the non-overlap between each machine and all obstacles. Other constraints ensure that no machine is assigned within the aisle.

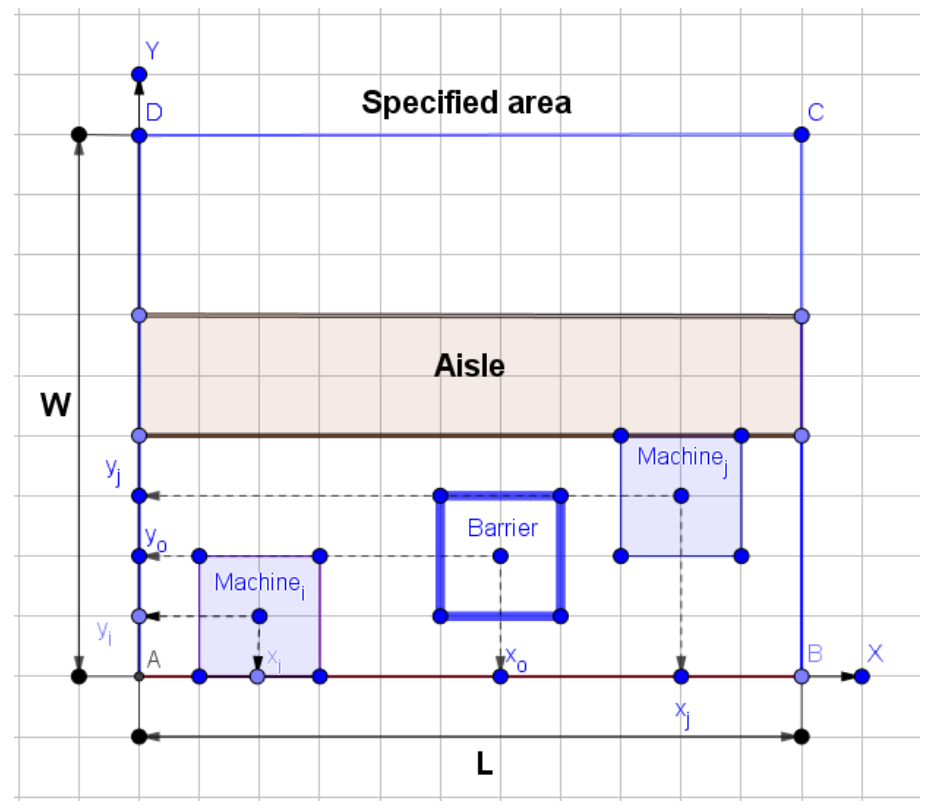

Figure1. Representation of the layout problem

\section{AN OVERVIEW OF OUR PROPOSED APPROACH}

As shown in Figure 2, our proposed approach is based on the combination of the genetic algorithm with $\mathrm{A}^{*}$ search algorithm. Genetic algorithm is used to generate various candidates' layouts. A* algorithm is used to determine the shortest path between two equipment is taking into account the different inner structure. In the following sections, a definition of the different specifications of each algorithm is introduced. 


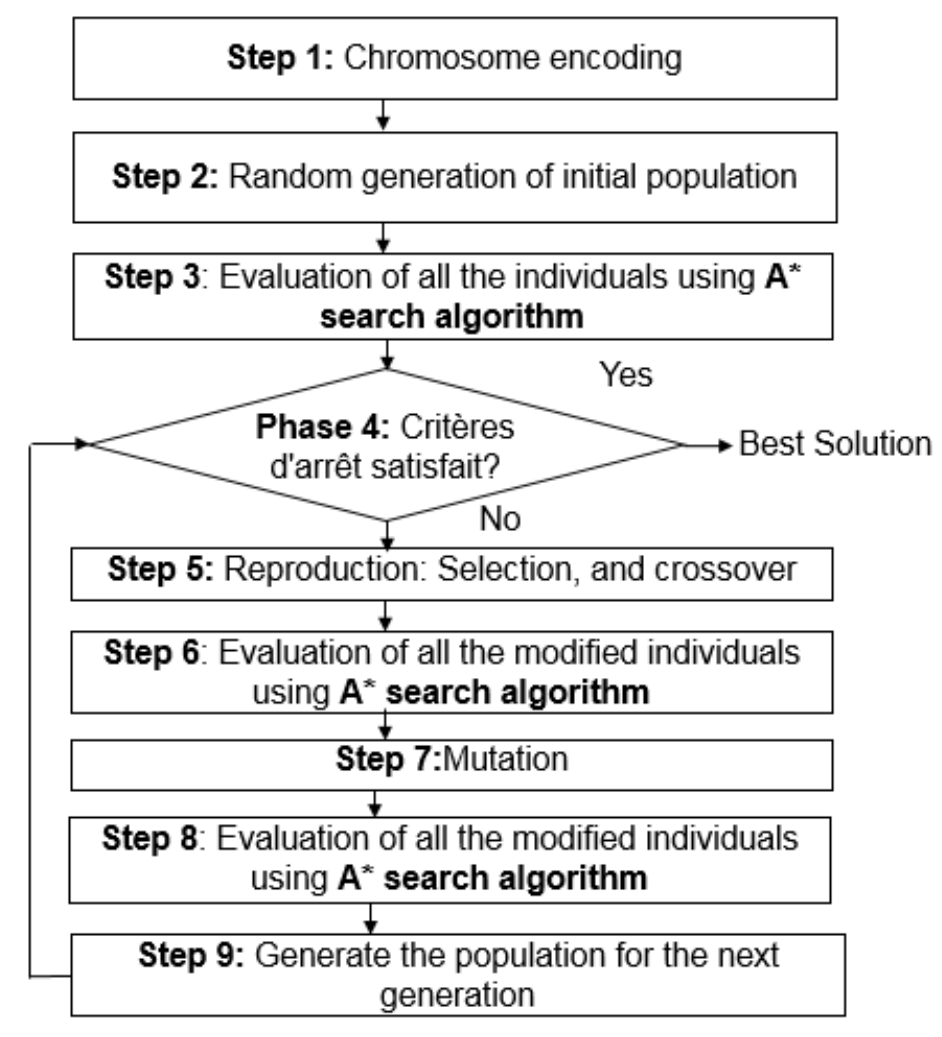

Figure2. Our proposed approach

\subsection{Overview of genetic algorithms (GAs)}

Genetic algorithm is a random search algorithm developed by Holland (1975). Genetic algorithms operate with populations, which are named chromosomes and encoded solutions of the problem [Mazinani et al. 2013]. The initial population is randomly generated and at each iteration, the population is updating by producing new individuals which replace some of the old ones. The reproduction uses a crossing and mutation process to create new ones. In our case, the evolution process is terminated when maximum number of iterations is attained. Crossover operators exchange the proprieties of two parents to create two new offspring. Mutation operators integrate new genetic structures in the population by simple modification applied to an individual. A selection strategy is applied to select best individuals to participate to crossover and mutation process.

Generation of the initial population

\section{Evaluation of initial individuals}

Selection of parents

Crossover process

Crossover probability $\left(\boldsymbol{P}_{c}\right)$

Crossover operator
The initial-solution individuals are randomly generated.

Each solution represents the coordinates $\left(x_{i}, y_{i}\right)$ of the centroid of each machine in the workshop. The length of the chromosome corresponds to the number of machines.

Evaluation of the transportation costs associated to the shortest distances between facilities.Erreur ! Source du renvoi introuvable.

Roulette wheel and tournament selection method.

A normal distribution with $\mu=0.7$ and $\sigma=0.1$.

One point crossover, two point crossover, three point 
crossover, and four point crossover

Mutation process

Mutation probability

Mutation operator

Evaluation of the children fitness value

Stopping criteria
A normal distribution with $\mu=0.18$ and $\sigma=0.06$.

Exchange and inversion operator

The elitist strategy

130 iterations

\subsection{Overview of $A^{*}$ search algorithm}

The $\mathrm{A}^{*}$ algorithm attempts to determine the shortest path between two machines. It starts by making the starting node in the open list. The open list contains the list of nodes to be explored. The starting node move from the open list to another list named closed list after focusing on its entire adjacent squares. The closed list includes all the explored nodes. A node must first pass through the open list before moving to the closed list. At the beginning, we must looking at neighboring nodes of the starting node while neglecting nodes with obstacles. These explored nodes are also make in the open list. For each of these nodes, the current node is considered as its 'parent square'. To determine if a node belong to the solution path its quality must be assessed. Three function are used in $A^{*}$ algorithm "f, $g$ and h" to calculate the lowest cost path to go from the start node to a goal node where $\mathrm{g}(\mathrm{x})$ represent the cost to go from starting node to the considered node, $\mathrm{h}(\mathrm{x})$ determines the cost from the considered node to the goal node. Hence, the search cost $f(x)$ is $f(x)=h(x)+g(x)$. This operation will be repeated when the destination node is reached.

\section{NUMERICAL RESULTS}

To evaluate the performance of the proposed methodology, we use an illustrative case inspired by the industrial environment. The problem has eight facilities, which are to be placed in an area of $30 * 20$ square surface units. An aisle having the same length as the workshop and two different vertical dimensions ( $y_{\text {lower }}=9$ and $y_{\text {upper }}=12$ ) was considered. The quantity of transported material between two machines is given in Table 1 . The input data about inner structures are reported in Table 2. Afterwards, a sensitivity analysis is made using Monte Carlo simulations to study the effects of various parameters on the transportation cost. Two sets of experiments are conducted in this work. In the first set of tests, the roulette (wheel selection) operator is used to select parents in order to create better offspring. The second set of tests is done with the tournament operator.

Table 1.Quantity of material flow from machine i to machine $\mathrm{j}$ for 8 facilities

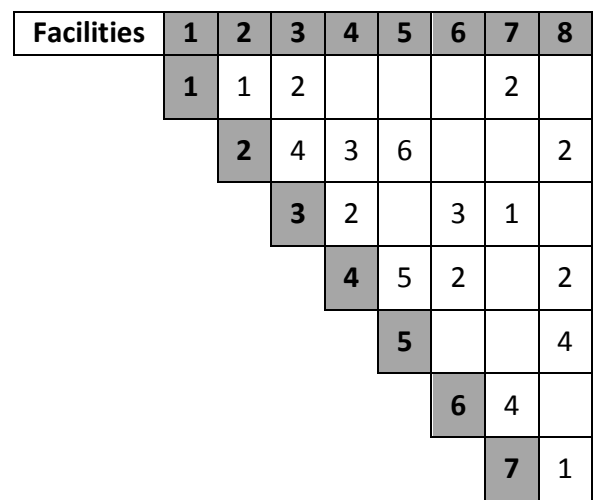


Table 2. Input data of obstacles

\begin{tabular}{lccc} 
& Coordinates (x,y) & Width & Length \\
\cline { 2 - 4 } Obstacle 1 & $(12,3)$ & 2 & 4 \\
Obstacle 2 & $(7,18)$ & 4 & 2
\end{tabular}

Discussion of the obtained results:

Regarding all the experimentations performed over 100 simulations; the combination of GA parameters that produces the best results is the following:

- Selection operator is tournament

- Population size is 194

- Crossover probability is 0.8049

- Mutation probability is 0.1024
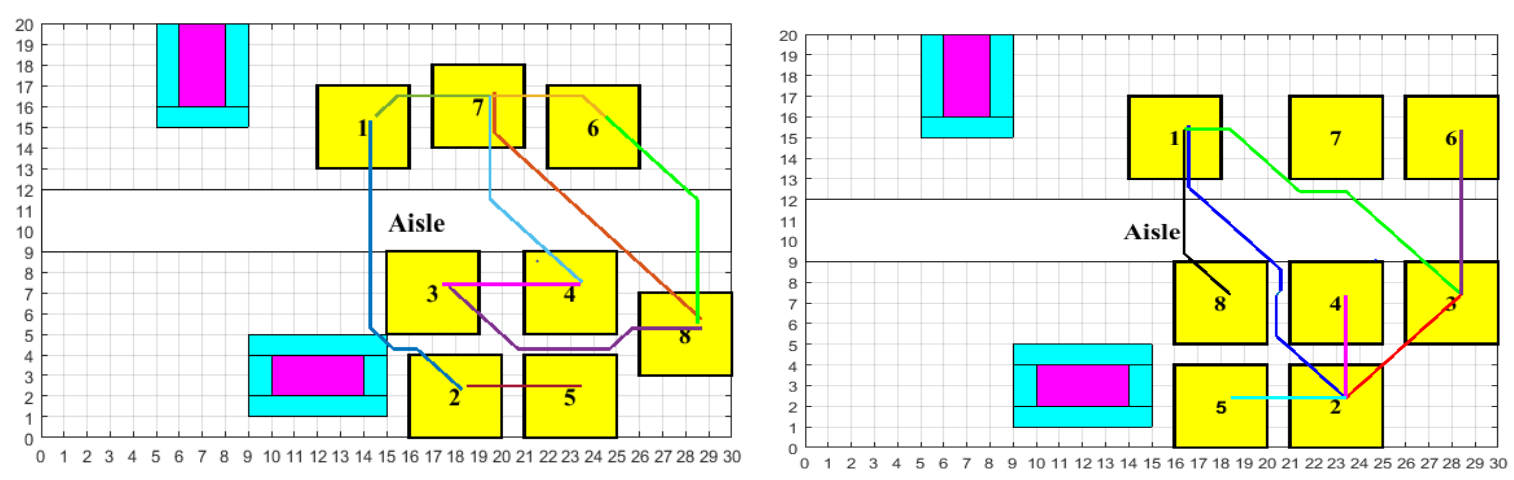

Figure.3 Monte Carlo simulations and product flow obtained by A* algorithm: best configurations founded via roulette wheel selection method and tournament method

The best layout show the practicability of the proposed method. Further, they show that the suggested method is efficient for the arrangement of a set of rectangular machines within a planar site without overlapping respecting a set of others constraints. The main advantage of the suggested approach is its ability to explore a large space of solutions, by considering more realistic distances between the machines. As illustrated in Fig.3, the different machines are concentrated in a specific zone, which allows companies to invest more for other projects.

\section{CONCLUSION}

This paper provides a new approach to find the most efficient layout in a given area. The main idea is to place machines in a restricted area while taking into consideration several kinds of geometric facility requirements. The objective function is to minimise the MHC which is associated to distance between different machines. A GA integrated with $\mathrm{A}^{*}$ allows to explore the solution space. The $A^{*}$ algorithm allows to determine the shortest distance between two machines. The GA is used to generate different layout over a set of iterations. The proposed algorithm parameters are calibrated using Monte Carlo simulations. Our proposed approach is applied on an illustrative case and the obtained results demonstrate the applicability of the proposed model. For future study, authors aims to generalize the formulation of the problem by considering various shape of machines as well as of plant floor.

\section{References}


[1] Tarkesh, H., Atighehchian, A., \& Nookabadi, A. S. (2009). Facility layout design using virtual multi-agent system. Journal of Intelligent Manufacturing, 20, 347-357.

[2] Ahmadi, A, Pishvaee, M.S., \& Akbari Jokar, M.R. (2017). A survey on multi-floor facility layout problems. Journal of Computers and industrial engineering, 107(2017), 158-170.

[3] Tompkins, J.A., White, J. A., Bozer, Y.A., \& Tanchoco, J. M. A. (2010). Facilities Planning. New York: Wiley.

[4] Chraibi, A. (2015). Outils d'aide à la décision pour la conception des blocs opératoires (Doctoral dissertation, Université Jean Monnet-Saint-Etienne).

[5] Drira, A., Pierrev, H., \& Hajri-Gabouj, S. (2007). Facility layout problems: A survey. Annual Reviews in Control, 31(2), 255-267.

[6] Che, A., Zhang, Y., \& Feng, J. (2017). Bi-objective optimization for multi-floor facility layout problem with fixed inner configuration and room adjacency constraints. Computers \& Industrial Engineering, 105, 265-276.

[7] Azadeh, A., Moghaddam, M., Nazari, T., \& Sheikhalishahi, M. (2016) Optimization of facility layout design with ambiguity by an efficient fuzzy multivariate approach. The International Journal of Advanced Manufacturing Technology,84(1), 565-579.

[8] Moslemipour, G., \& Lee, T. (2012). Intelligent design of a dynamic machine layout in uncertain environment of flexible manufacturing systems. Journal of Intelligent Manufacturing, 23, 1849-1860.

[9] Ahmadi, A., \& Akbari Jokar, M. R. (2016). An efficient multiple-stage mathematical programming method for advanced single and multi-floor facility layout problems. Journal of applied Mathematical Modelling, 40(9-10), 5605-5620.

[10] Kundu, A., \& Dan, P.K. (2012). Metaheuristic in facility layout problems: current trend and future direction. International Journal of Industrial and Systems Engineering, 10(2), $238-253$.

[11] Gonçalves, J.F., \& Resende, M.G. (2015). A biased random-key genetic algorithm for the unequal area facility layout problem. European Journal of Operational Research, 246(1), 86-107.

[12] Xie, Y., Zhou, S.,Xiao, Y., Kulturel-Konak, S., \& Konak, A. (2018). A $\beta$-accurate linearization method of Euclidean distance for the facility layout problem with heterogeneous distance metrics. European Journal of Operational Research, 265,26-38.

[13] Xie, Y., Zhou, S.,Xiao, Y., Kulturel-Konak, S., \& Konak, A. (2018). A $\beta$-accurate linearization method of Euclidean distance for the facility layout problem with heterogeneous distance metrics. European Journal of Operational Research, 265,26-38.

[14] Holland, J.H. (1975). Adaptation in natural and artificial systems: an introductory analysis with applications to biology, control, and artificial intelligence. Michigan Press.

[15] Mazinani, M., Abedzadeh M., \& Mohebali N. (2012). Dynamic facility layout problem based on flexible bay structure and solving by genetic algorithm. The International Journal of Advanced Manufacturing Technology, 65(5-8), 929-943. 
